\title{
Doing Critical Health Communication: Negotiating the Terrain of Transdisciplinary Collaboration
}

\author{
Kimberly N. Kline* and Shamshad Khan \\ Department of Communication, University of Texas at San Antonio, San Antonio, TX, United States
}

In this reflective article, drawing on our personal and productive experiences with transdisciplinary research, we think about how critical health communication scholars can speak to audiences outside the discipline of communication in order to make an impact on public health and policy, health promotion, and health care delivery. We first take into consideration how we are situated in our relationship with transdisciplinary research, as well as the challenges and opportunities involved in collaborating with transdisciplinary teams. We then discuss ways we can navigate the inherent method/ological tensions in such collaborations. We argue that while the multidimensional nature of health and illness-especially in the face of skyrocketing

\section{OPEN ACCESS}

Edited by:

Shaunak Sastry,

University of Cincinnati, United States

Reviewed by:

Peter Johannes Schulz,

University of Lugano, Switzerland

Jill Yamasaki,

University of Houston, United States

*Correspondence:

Kimberly N. Kline

kim.kline@utsa.edu

Specialty section: This article was submitted to Health Communication, a section of the journal Frontiers in Communication

Received: 16 May 2019 Accepted: 30 August 2019 Published: 18 October 2019

Citation:

Kline KN and Khan S (2019) Doing Critical Health Communication:

Negotiating the Terrain of

Transdisciplinary Collaboration.

Front. Commun. 4:51.

doi: 10.3389/fcomm.2019.00051 healthcare costs and disparities - mandates transdisciplinary research and action, navigating the epistemic and methodological boundaries is nevertheless not easy. Here, we focus on how the methodological considerations of "critical" health scholarship are situated vis-à-vis the epistemic commitments in the disciplines of our potential allies and whether it is possible to collaborate in ways that can enhance the goal of social justice, equity and human rights within public health and communication.

Keywords: critical health communication, paradigms, research methods, transdisciplinary teams, rigor and bias in research

Critical approaches to health communication are concerned with how power influences society's "cultural constructions of health and responses to illness" (Zoller, 2014, p. 270). Critical scholars are motivated by an explicitly political and ethically grounded goal of fostering social justice, equity and human rights, achieved by unmasking the sociopolitical forces that regulate and constrain the health and illness experiences of various disadvantaged, marginalized, and/or oppressed groups of people. As Zoller and Kline (2008) explain, "Critical theorizing involves deconstructing dominant, taken-for-granted assumptions about health, often with the hope of introducing possibilities for alternative, more inclusive meaning systems" (p. 271). One might say critical approaches are inherently-indeed, overtly_ "biased." That is, critical health communication scholars enter into the fray with a clear personal commitment to promote progressive social change as a lever for health and development.

Critical approaches presume hegemonic structures and, importantly, the need to rectify concomitant social inequities and injustices that impact an individual's lived experiences of health and well-being. For instance, Dutta's (2008) influential culture-centered approach to health communication recognizes that certain sectors of the population have been marginalized by "institutional practices of policymakers, interventionists, and program evaluators" and the goal of 
critical research is to aid them in resisting and navigating a system that continues to locate them on the margins of society (Culture Centered Approach, 2017). Likewise, while feminism is not a singular belief or political position, two points of consensus are that (1) gender is a "key organizer of social life" and (2) we must take action to make our social life more equitable (Sprague, 2016, p. 3).

In other words, in critical scholarship, the stance, presumption or "bias" against hegemonic structures and in support of giving voice to and empowering those who are marginalized is acknowledged and embraced, explicit and intentional. Yet, much of the discussion about ensuring the quality and/or integrity of academic research is concerned with reducing, controlling for, and eliminating bias or what many within the social scientific tradition see as the "problematic" of subjectivity in research. Couched in terms of "rigor" (discussed below), this concern is the hallmark of traditional quantitative methods; yet debates about qualitative research methods also invoke misgivings about bias when it comes to all stages of inquiry (for discussion of debate see Grbich, 1999).

The focus on "bias" in research is troubling for critical health communication scholars because in order to have the most impact we must go beyond the realm of our own critical scholarship to collaborate with scholars and practitioners who tend to favor (social) scientific method/ologies-i.e., those that eschew "bias." If we want to positively impact the everyday lived experiences of people, it is incumbent upon us to collaborate and forge partnerships with those on the front lines, including scholars and professionals in public health and nursing, medical education and training, health education and health promotion, health psychology and sociology, and other health professional settings (Kreps, 2012). As Kotowski and Miller (2010) explain, it is through this transdisciplinary collaboration that we can "grow the field beyond its walls in the academy, increase[] its already sizable impact and help[] it mature as an important area of research into human communication processes within the health domain" (Kotowski and Miller, 2010, p. 567; see also Kreps and Maibach, 2008).

Health communication is fundamentally an applied discipline (Zoller, 2014); likewise, while not all critical research in health communication may be outright "applied," it must always be "applicable"-even as a practice of critique. It is often through such exercise (e.g., the active process of deconstruction) that alternative views of the world, innovative approaches to health and wellness, and progressive social change become possible. Ultimately, the goal is to amplify our scholarly voices and make greater impact by finding common ground, both within and outside the discipline of communication. In this way, critical health communication scholars add value to public health and policy debates and interventions through their continued commitment to praxis (Zoller, 2014).

In this article, we discuss the challenges and opportunities critical health communication scholars face when speaking to audiences outside the communication discipline in attempts to make an impact on public health and policy, health promotion, and health care delivery. In other words, how are we situated with regard to transdisciplinary research? How do we make the most of opportunities for collaborative research and how do we navigate the inherent tensions in such collaborations? In particular, we discuss how the methodological considerations of "critical" health scholarship are situated vis-à-vis the epistemic commitments in the disciplines of our potential allies.

\section{METHOD/OLOGICAL DISCONNECTS}

It is always a challenge to discuss the implications of paradigmatic differences without seeming to take and "us vs. them" attitude. Still, the only path to productive dialogue lies in acknowledging the sources of our tensions, recognizing the contributions of alternative perspectives, and negotiating acceptable compromises.

Thus, while thinking about the ways in which critical health communication scholars may contribute to or form partnerships with transdisciplinary research teams, we need to realize that the path is not that easy and rather fraught with multiple challenges. For instance, in his work with transdisciplinary team members including physicians, anthropologists, demographers, public health researchers and managers (Lorway et al., 2017; Khan et al., 2018; Huynh et al., 2019), Khan and colleagues found it productive but also challenging to use critical concepts. Consider a concept like "structural violence": because it refers to large-scale social and structural processes, as well as multiple and intersecting layers of disease causation, for example, it is both virtually invisible and hard to quantify. Even when an argument along this line sounds convincing, a critical scholar is often asked, "where is the evidence?" and "how do you prove it?" Transdisciplinary teams may come to embrace critical concepts-indeed, in the past 6 years, Khan and colleagues have implemented multiple research and intervention projects that address structural drivers of HIV.

We believe that a significant struggle in establishing these collaborative relationships stems from the disjuncture in the method/ological commitments of critical scholars and our potential collaborators. These challenges to critical research are captured in the critiques of qualitative methods ${ }^{1}$ that are seen as inherently "biased" in that they bring elements of social constructionism in approaches to knowledge and understanding of human experience.

We want to clarify that we are not trying to conflate qualitative research methods with critical approaches. Zoller and Kline (2008) emphasize that "interpretive/critical approaches may seem synonymous with qualitative methodologies (and, alternatively, post-positivist approaches synonymous with quantitative methodologies); in research practice, however, they are not always the same" (p. 95). We start with this caveat because critical approaches are not and do not employ a specific method. As Sprague explains (2016), "We can gather information by listening, watching, and examining documents; we organize our

\footnotetext{
${ }^{1}$ As McDonald (2017) explains, "Critical methods have much in common with qualitative methods, as critical researchers collect primarily qualitative data, take a subjective approach to research, recognize that knowledge is limited and partial, and seek to build connections with rather than distance themselves from research participants."
} 
observations by counting instances of preconceived categories and/or by looking for unanticipated patterns" (p. 5). However, as Sprague's book Feminist Methodologies for Critical Researchers cogently demonstrates, critical scholars can certainly employ quantitative methods. Others have also discussed the use of subversive (Denzin and Lincoln, 2018) or transgressive (Lincoln et al., 2018) statistics.

This is an important distinction since recently there has been an attempt to bring quantitative research or mixed methods within critical health communication research (e.g., Thaker et al., 2018, 2019). In fact, critical scholars are generally good at drawing on and developing conceptual categories, many of which (e.g., "social capital," or "structural violence") are now used or "applied" by social scientific scholars with the help of quantifiable indicators to facilitate application and testing in intervention planning. Not surprisingly, at the 2018 National Communication Association (NCA) panel entitled "Doing Critical Health Communication: Playing with Methods," several scholars acknowledged the need and importance of using quantitative and/or mixed methods in critical research in order to speak to wider audiences including policy makers who are persuaded by quantitative data. However, participants and panel members also commented on struggling against the dominant positioning around what counts as "credible" and "reliable" data. The point is that it is certainly possible and productive for critical health communication scholars to employ quantitative methods in their research-a method that speaks to our more quantitatively aligned allies.

Nevertheless, the tension between qualitative and quantitative methods remains present and is most likely a function of ongoing paradigmatic differences or conflict. That is, "methods" are traditionally presumed to have consistent and competing methodological principles, values, etc. A methodology is a "researchers' choices for how to use these methods" and "each methodology is founded on either explicit or, more often, unexamined assumptions about what knowledge is and how knowing is best accomplished" (Sprague, 2016, p. 5). More to the point, given their contrasting (some would say incompatible) metatheoretical (ontological, epistemological, axiological) assumptions, methodologies aligned with qualitative and quantitative methods have historically been at odds (Zoller and Kline, 2008).

The distinction between these paradigmatic commitments and the accompanying debates regarding (in)commensurability are well-documented (Grbich, 1999; Denzin and Lincoln, 2018; Lincoln et al., 2018). Thus, here we briefly summarize the connection to so-called "bias." (Post)positivism is a methodological approach that believes in and privileges "objectivity" or the idea "that if, and only if, we systematically and dispassionately observe the data of the empirical world, we can detect the lawful patterns of which they are evidence" (Sprague, 2016, p. 35). The assumption is that "'truth' can transcend opinion and personal bias" (Denzin and Lincoln, 2018 , p. 8) and, thus, method/ologies focus on procedures "as devoid as instrumentally possible of human bias, misperception, and other 'idols"' (Lincoln et al., 2018, p. 135). Alternatively, critical methodologies embrace "subjectivity" starting with the "most basic ontological assumption that our perceptions of reality are constituted as subjects attach meaning to phenomena and that these meanings arise through interactions [and the] concomitant epistemological assumption... that we come to agreement about what is real intersubjectively" (Zoller and Kline, 2008, p. 93). In other words, "knowledge is not and cannot be objective because values are embedded into the very definition of what counts as knowledge" (McDonald, 2017, p. 3 ). As we articulated in the introduction, critical methodologies always already presume the personal stance of the critical scholar against social inequities and in favor of social justice. It would seem, then, that post-positivist ontological and epistemological assumptions are diametrically opposed to those associated with critical approaches to knowledge and understanding.

We should acknowledge here that we have been to some extent using the term "bias" ironically given that even commonuse definitions treat "bias" as pejorative: "a particular tendency, trend, inclination, or opinion, especially one that is preconceived or unreasoned" (dictionary.com, our emphasis). When talking about academic research, the term "biased" is essentially code for overly "subjective" and set in contradistinction to the concept of “objectivity." As Lincoln et al. (2018) point out, bias "relate[s] directly to the concerns of objectivity that flow from positivist inquiry [and] are reflective of inquirer blindness or subjectivity" (p. 140). Thus, as in the common-use of "bias," in (post-positivist) scholarly use "bias" is also a pejorative and the influence of subjectivity is inherently called into question. Post-positivists believe that "subjectivity is an obstacle to knowledge" (Sprague, 2016), "is too closely aligned with the personal agenda of the researcher(s)" and "provides a distortion in the results of a study" (Galdas, 2017, p.1); it "is thought to destabilize objectivity and introduce subjectivity" (Lincoln et al., 2018, p. 140) which, in turn, can be seen as a "human contamination" (Denzin and Lincoln, 2018, p.35) (all our emphases). We, of course, are not using the concept of "bias" as a pejorative!

Most critical scholars would not necessarily see a problem with presence of "biases" or values in research since the assumption is that they are always present regardless of what kind of research one does. In short, there is no such thing as value-free science. Instead, critical scholars want these biases or values to be publicly debated so we can see whose biases and values are present and what can be done to change the statusquo (Lupton, 1994). For example, in her beautifully written book, Hidden Arguments: Political Ideology and Disease Prevention Policy, Tesh (1988) states: "I argue not that values be excised from science and from policy but that their inevitable presence be revealed and their worth be publicly discussed" (p.3). Packed into this single sentence is not only her attempt to problematize the ideologically rich slogan of "neutrality" in science and public policy, but also her insistence on the need to publicly debate the hidden arguments and values which are often more fundamental.

To be fair, we should also concede that whether "bias" amounts to an unreasonable prejudice partly depends on the eye of the beholder. Zoller and Kline (2008) critique a number of "biases" in health communication research that stem from post-positivist commitments. The problem as we see is that in discussions of research rigor, "bias" is treated as synonymous with 
subjectivity. And, of course, the privileging of "subjectivity" is one of the hallmarks of critical scholarship. So, how do we bridge this major gap or chasm in our approaches to research that can potentially lead to meaningful, transdisciplinary collaborations?

\section{METHOD/OLOGICAL NEGOTIATIONS}

Our transdisciplinary colleagues have already begun to find value in the contributions of critical scholars (Padgett and Henwood, 2009). Like Khan, Kline has had a long, productive, and funded collaboration with research teams that include epidemiologists, public health professionals, and physicians (Rustveld et al., 2009; Eberth et al., 2014; Kline et al., 2016). Indeed, chances of receiving government-sponsored and/or large-scale funding may be higher if we use mixed methods and have a transdisciplinary team of scholars on board (Darbyshire, 2004; Padgett and Henwood, 2009; Treise et al., 2016). The challenge is to actively work at keeping the conversation going and to persevere in mainstreaming collaborative efforts.

One suggestion for facilitating collaboration between paradigmatically different scholars comes down to adjusting qualitative methods to fit within the dominant post-positivist discourse on methodology, possibly by recuperating objectivist terminologies to justify the "rigor" of qualitative methods. For instance, in her often cited article (with more than 5,000 citations according to Google Scholar), renowned qualitative scholar and professor of nursing Morse et al. (2002) contends that qualitative scholars should return to using the terms "validity," "generalizability, "reliability," and "objectivity" rather than using Lincoln and Guba's (1985; see also Guba, 1981) terminology of "credibility," "transferability," "dependability," and "confirmability." More recently, reiterating this appeal, she elaborates that we should "develop, refine, and test analytic processes and strategies that fit qualitative inquiry while also remaining consistent with concepts used by the larger social science community" and maintains that "only then will we be able to describe our methods in a way that other social sciences will comprehend and respect our research" (Morse, 2015, p. 1,220; our emphasis).

Morse's discussion about methodological strategies for ensuring rigor (or in Guba and Lincoln's terminology, trustworthiness) in qualitative research provides valuable insight, but one has to wonder if a return to the language of objectivity would be consistent with the goals of critical scholarship. Would doing so be an invitation to evaluate critical qualitative research using traditional quantitative criteria? Would "scientists" understand Morse's nuanced reframing of these terms to accommodate (interpretive and) critical scholarship? We expect that regardless of how carefully delineated the use of these terms in the context of critical, qualitative research, the terms "validity" and "reliability" would inevitably invoke the idea of value-free scientific neutrality. At best, defending new "definitions" or applications of old terms would place critical scholars in the tenuous position of provoking readers by reopening wounds of paradigmatic frictions. Perhaps more unsettling, critical scholars would be left with the responsibility of constantly reiterating method/ological criteria that conflict with their foundational principles. That is, reversion to post-positive terminology potentially undermines attempts to challenge hegemonic postpositivist epistemologies. For instance, Maori and postcolonial scholar Smith (2012) vigorously critiques academic research and methods-mostly social scientific-that have historically been complicit in the project of imperialism by privileging Euro-centric and exclusionary ways of knowing and "discovering truth," thereby hierarchizing knowledge and delegitimizing indigenous ways of knowing and being.

As Bochner (2018) elucidates, "The trouble with inherited words like 'rigor' [or related "reliability" and "validity," etc.] is that they impede our use of other words that better express the beliefs, goals, and standards of the members of our community's way of life" (p. 361). Frankly, solutions such as returning to post-positive terminologies (and embedded values) in order to reconcile paradigmatic differences or to bolster the credibility of our research is essentially an entreaty for critical scholars to adapt to post-positivistic standards and norms. Where, then, is the middle-ground, substantive and healthy dialogue between disciplines?

Alternatively, Grbich (1999) attempts to bridge the gap between "those who have emphasized the importance of rigorous qualitative research and those who regard 'rigor' as inappropriate" by defining rigor as "the researcher's attempt to use as tight a research design as possible" (p. 61). Although Grbich does not delineate what she means by "tight" design, we read it as research design that is actively transparent, intentionally descriptive, and logically consistent. Perhaps most important, the methodological steps and the rationale behind are meticulously established and articulated before the scholar analyzes the "data."

\section{METHOD/OLOGICAL VERACITY}

The critical stance we have toward research-the presence of values and concern with sources of knowledge and knowledge production and their role in changing or perpetuating social and material contexts of health and illness-creates challenges but, hopefully, also opportunities. That is, we can find a way to speak to our potential colleagues and allies in ways that are convincing and yet maintain our commitment as critical scholars.

Let us first acknowledge that there is a distinction to be made between weak scholarship and inherent flaws with method/ologies. For instance, given the subjective nature of qualitative method/ologies, some may be concerned that qualitative researchers may try to defend interpretations by using only those examples that support the researcher's a priori assumptions (i.e., "biases"). Yet, that would be similar to the assertion that because some quantitative scholars have tried to generalize their findings based a nonprobability sampling strategy, all quantitative scholarship is suspect. Indeed, as Padgett (2012) reminds us, "it is fair to say that generalizability is often a problem in quantitative studies because many are unable to meet the assumptions of random sampling, normal distributions, and bounded sampling frames that underlie inferential statistics" (p. 4). 
There are any number of textbooks and published studies that address the details of how to ensure the quality of qualitative research-far too many to cite here (though we've referenced some throughout this article). In addition, there are many checklists available for assessing the quality of research (see Majid and Vanstone, 2018 for a list of over 100 checklists). However, we believe that lynchpin to achieving respect and consideration for our method/ologies lies in being self-reflective about our own biases and assumptions, and transparency with regard to how they impinge on the method/ological choices we make in our research (Galdas, 2017).

Both critical reflexivity and attention to positionality are closely connected and are routinely used (or should be used) by critical scholars to interrogate their assumptions and biases (as they plan and conduct their research), including their own power positions in relation to the subject of study. This constant process of critical engagement with personal values, biases and power positions and bringing them forward for the audience to see and examine is a unique and cutting-edge contribution that critical scholars make to the world of research and methods. Indeed, in the process of doing so, they necessarily go beyond reifying methods and tools where everything including one's own biases and assumptions are open for scrutiny.

As important as the need for self-reflexivity, it is also the case that to be taken seriously outside the domain of critical theorizing in health communication scholarship, we need to attend carefully to describing and explaining our methods-i.e., the tools and conceptual categories that we use, the rationale for using them, and the contribution that they make to public health and policy and society at large (e.g., Dutta-Bergman, 2004; Zoller, 2005; Kline, 2007; Dutta and Basu, 2008; Basu, 2011; Khan, 2014; Agarwal, 2018; Khan et al., 2018).

It may seem as if critical (and qualitative scholars) are always called on to "prove" the validity of their work, but there is still a tendency among some to ignore attention to details in describing

\section{REFERENCES}

Agarwal, V. (2018). Taking care, bringing life: a post-structuralist feminist analysis of maternal discourses of mothers and dais in India. Health Commun. 33, 423-432. doi: 10.1080/10410236.2016.1278492

Basu, A. (2011). HIV/AIDS and subaltern autonomous rationality: a call to recenter health communication in marginalized sex worker spaces. Commun. Monogr. 78, 391-408. doi: 10.1080/03637751.2011.589457

Bochner, A. P. (2018). Unfurling rigor: on continuity and change in qualitative inquiry. Qualit. Inquiry, 24, 359-368. doi: 10.1177/1077800417727766

Culture Centered Approach (CCA) (2017). What is CCA. Available online at: from http://www.culturecenteredapproach.com/index.php/what-is-cca. (accessed October, 2018).

Darbyshire, P. (2004). Qualitative research in the grant-funding jungle. Collegian 11, 8-11. doi: 10.1016/S1322-7696(08)60459-X

Denzin, N. K., and Lincoln, Y. S. (2018). "Introduction: the discipline and practice of qualitative research," in The Sage Handboook of Qualitative Research, 5th ed. eds N. K. Denzin and Y. S. Lincoln (Thousand Oaks, CA: Sage), 1-26.

Dutta, M. J. (2008). Communicating Health: A Culture-Centered Perspective. London: Polity.

Dutta, M. J., and Basu, A. (2008). Meanings of health: interrogating structure and culture. Health Commun. 23, 560-572. doi: 10.1080/10410230802465266 and, to some extent, following a systematic approach to methods. Some critical scholars, in the spirit of their critical tradition, find methods and especially the need for attention to details in describing them, rather constricting and limiting. In other words, their concern is that structured methods, by virtue of being "disciplining" in nature, run the risk of blunting critical thinking and creative ideas. Padgett (2012) recognizes that while offering a clear rationale for using qualitative methods "may seem to be an unfair burden (quantitative researchers need not do this), it is in fact an opportunity to educate the reader and convey a sense of mastery" (p. 208). As she also points out, "When the topic is appropriate, this is an easy argument to make" (p. 208). More to the point, being explicit in our methodological details helps in addressing questions, challenges, and/or suspicions head-on.

\section{CONCLUSION}

Critical health communication scholars are at an important juncture. Having made significant impact within the field of health communication, we are poised to work alongside our transdisciplinary colleagues. Given the acceleration of health inequities in the world and promises and opportunities of doing collaborative research across the globe, we must be bold and committed to moving forward on this path toward transdisciplinary research. The path of collaborative research is not easy, but opportunities exist if we are willing to speak to audiences outside our domain as well as to the new generations of critical health communication scholars who will lead future transdisciplinary teams.

\section{AUTHOR CONTRIBUTIONS}

Both authors have made a substantial, direct and intellectual contribution to the work, and approved it for publication.

Dutta-Bergman, M. (2004). Poverty, structural barriers, and health: a Santali narrative of health communication. Qualit. Health Res. 14, 1107-1123. doi: $10.1177 / 1049732304267763$

Eberth, J. M., Kline, K. N., Moskowitz, D. A., Montealegre, J. R., and Scheurer, M. E. (2014). The role of media and the Internet on vaccine adverse event reporting: a case study of human papillomavirus vaccination. J. Adolescent Health 54, 289-295. doi: 10.1016/j.jadohealth.2013.09.005

Galdas, P. (2017). Revisiting bias in qualitative research: reflections on its relationship with funding and impact. Int. J. Qualit. Methods 16, 1-2. doi: $10.1177 / 1609406917748992$

Grbich, C. (1999). Qualitative Research in Health. Thousand Oaks, CA: Sage.

Guba, E. (1981). Criteria for assessing the trustworthiness of naturalistic inquiries. Educ. Commun. Technol. J. 29, 75-91.

Huynh, A., Khan, S., Nair, S., Chevrier, C., Roger, K., and Isac, S., Lorway, R. (2019). Intervening in masculinity: work, relationships and violence among the intimate partners of female sex workers in South India. Crit. Public Health 29, 156-167. doi: 10.1080/09581596.2018.1444266

Khan, S. (2014). Manufacturing consent?: media messages in the mobilization against HIV/AIDS in India and lessons for health communication. Health Commun. 29, 288-298. doi: 10.1080/10410236.2012.753139

Khan, S., Lorway, R., Chevrier, C., Dutta, S., Ramanaik, S., and Roy, A., Becker, M. (2018). Dutiful daughters: HIV/AIDS, moral pragmatics, female citizenship 
and structural violence among Devadasis in northern Karnataka, India. Global Public Health 13, 1065-1080. doi: 10.1080/17441692.2017.1280070

Kline, K. N. (2007). Cultural sensitivity and health promotion: assessing breast cancer education pamphlets designed for African American women. Health Commun. 21, 85-96. doi: 10.1080/10410230701283454

Kline, K. N., Montealegre, J. R., Rustveld, L. O., Glover, T. L., Chauca, G., Reed, B. C., et al. (2016). Incorporating cultural sensitivity into interactive entertainment-education for diabetes self-management designed for Hispanic audiences. J. Health Commun. 21, 658-668. doi: 10.1080/10810730.2016.1153758

Kotowski, M. R., and Miller, L. E. (2010). The impact of interdisciplinary collaboration. Health Commun. 25, 567-568. doi: $10.1080 / 10410236.2010 .496718$

Kreps, G. L. (2012). Translating health communication research into practice: the importance of implementing and sustaining evidence-based health communication interventions. Atlantic J. Commun. 20, 5-15. doi: $10.1080 / 15456870.2012 .637024$

Kreps, G. L., and Maibach, E. W. (2008). Transdisciplinary science: the nexus between communication and public health. J. Commun. 58, 732-748. doi: 10.1111/j.1460-2466.2008.00411.x

Lincoln, Y. S., and Guba, E. G. (1985). Naturalistic Inquiry. Beverly Hills, CA: Sage.

Lincoln, Y. S., Lynham, S. A., and Guba, E. G. (2018). "Paradigmatic controverses, contradictions, and emerging confluences, revisited," in The Sage Handboook of Qualitative Research, 5th ed. eds N. K. Denzin and Y. S. Lincoln (Los Angeles: Sage), 108-150.

Lorway, R., Khan, S., Chevrier, C., Huynh, A., Zhang, J., and Ma, X., Yu, N. (2017). Sex work in geographic perspective: a multi-disciplinary approach to mapping and understanding female sex work venues in Southwest China. Global Public Health 12, 545-564. doi: 10.1080/17441692.2015.1123748

Lupton, D. (1994). Toward the development of critical health communication praxis. Health Commun. 6, 55-67. doi: 10.1207/s15327027hc0601_4

Majid, U., and Vanstone, M. (2018). Appraising qualitative research for evidence syntheses: a compendium of quality appraisal tools. Qualit. Health Res. 28, 2115-2131. doi: 10.1177/1049732318785358

McDonald, J. (2017). "Critical methods," in The International Encyclopedia of Communication Research Methods, ed J. R. Matthes (Chichester: WileyBlackwell).

Morse, J. M. (2015). Critical analysis of strategies for determining rigor in qualitative inquiry. Qualit. Health Res. 25, 1212-1222. doi: $10.1177 / 1049732315588501$

Morse, J. M., Barrett, M., Mayan, M., Olson, K., and Spiers, J. (2002). Verification strategies for establishing reliability and validity in qualitative research. Int. J. Qualit. Methods 1, 13-22. doi: 10.1177/160940690200100202

Padgett, D. K. (2012). "Strategies for rigor," In Qualitative and Mixed Methods in Public Health. ed D. K. Padgett (Thousand Oaks, CA: Sage), 203-220.
Padgett, D. K., and Henwood, B. F. (2009). Obtaining large-scale funding for empowerment-oriented qualitative research: a report from personal experience. Qualit. Health Res. 19, 868-874. doi: 10.1177/10497323083 27815

Rustveld, L. O., Pavlik, V. N., Jibaja-Weiss, M. L., Kline, K. N., Gossey, T., and Volk, R. J. (2009). Adherence to diabetes self care behaviors in English and Spanish speaking Hispanic men. Patient Preference Adherence 3, 123-130. doi: 10.2147/PPA.S5383

Smith, L. (2012). Decolonizing Methodologies : Research and Indigenous peoples, 2nd ed. London: Zed Books.

Sprague, J. (2016). Feminist Methodologies for Critical Researchers: Bridging Differences, 2nd ed. Lanham, MD: Rowman \& Littlefield.

Tesh, S. (1988). Hidden Arguments : Political Ideology and Disease Prevention Policy. New Brunswick: Rutgers University Press.

Thaker, J., Dutta, M., Nair, V., and Rao, V. P. (2018). The interplay between stigma, collective efficacy, and advocacy communication among men who have sex with men and transgender females. J. Health Commun. 23, 614-623. doi: 10.1080/10810730.2018.1499833

Thaker, J., Dutta, M. J., Nair, V., and Rao, V. P. (2019). "Media portrayal stigma among gender and sexual minorities: Meaning, power, and resistance," in Communicating for Social Change: Meaning, Power, and Resistance, eds M. J. Dutta and D. B. Zapata (Palgrave Macmillan), 383-407.

Treise, D., Baralt, C., Birnbrauer, K., Krieger, J., and Neil, J. (2016). Establishing the need for health communication research: best practices model for building transdisciplinary collaborations. J. Appl. Commun. Res. 44, 194-198. doi: $10.1080 / 00909882.2016 .1155729$

Zoller, H. M. (2005). Women caught in the multi-causal web: a gendered analysis of Healthy People 2010. Commun. Stud. 56, 175-192. doi: 10.1080/00089570500078809

Zoller, H. M. (2014). "Critical approaches," in Encyclopedia of Health Communication, ed T. L. Thompson (Thousand Oaks, CA: Sage), 270-272.

Zoller, H. M., and Kline, K. N. (2008). Interpretive and critical research in health communication. Communication Yearbook 32, 88-135. doi: $10.1080 / 23808985.2008 .11679076$

Conflict of Interest: The authors declare that the research was conducted in the absence of any commercial or financial relationships that could be construed as a potential conflict of interest.

Copyright (C) 2019 Kline and Khan. This is an open-access article distributed under the terms of the Creative Commons Attribution License (CC BY). The use, distribution or reproduction in other forums is permitted, provided the original author(s) and the copyright owner(s) are credited and that the original publication in this journal is cited, in accordance with accepted academic practice. No use, distribution or reproduction is permitted which does not comply with these terms. 